\title{
ASYMPTOTIC STRESS FIELD AT TIP OF NOTCH TERMINATING TO INTERFACE
}

\author{
Grzegorz Mieczkowski ${ }^{1}$, Dariusz Szpica ${ }^{1}$, Andrzej Borawski ${ }^{1}$, \\ Mohamed M. Awad ${ }^{2}$, Ahmed Elgarayhi' ${ }^{2}$, Mohammed Sallah ${ }^{2}$ \\ ${ }^{1}$ Bialystok University of Technology, Poland; ${ }^{2}$ Mansoura University, Egypt \\ g.mieczkowski@pb.edu.pl,d.szpica@pb.edu.pl, a.borawski@pb.edu.pl,m_m_awad@mans.edu.eg, \\ elgarayhi@mans.edu.eg,msallahd@mans.edu.eg
}

\begin{abstract}
The paper presents the results of research related to the analytical and numerical description of the local stress fields generated by a sharp notch, which tip is located at the interface of the bi-material structure. While the problem of the crack initiated on the line separating two different elastic materials is quite well described in the literature, the problem of corners with a non-zero apex angle is insufficiently investigated. The analytical description of the stress fields around the corner apex, with the use of generalized stress intensity factor (GSIF), was obtained on the basis of the equations of the theory of elasticity and the Airy stress function. The analytical and numerical method was used to determine the value of GSIFs. Assuming that the specimens (single edgenotched plate) were subjected to uniaxial load, the GSIFs were determined. The influence of the notch height and angle on the value of generalized stress intensity factors was investigated. Moreover, the calculations were made for various relative stiffness (Young's moduli proportions of individual components of the bi-material structure). It was found that the normalized GSIF values increase with a decrease in the relative stiffness and an increase in the notch height and its apex angle. The results obtained, if possible, were compared with the literature data. A satisfactory agreement was found with the results presented by other scientists.
\end{abstract}

Keywords: composite, interface, V-notch.

\section{Introduction}

The intensive development of composite materials has been noticeable in recent years. Composites are usually anisotropic materials. They may have more or less a complex periodic structure and often containing voids, inclusions or other internal defects that cause a local stress concentration. The stress fields with high gradients consequently lead to the initiation of the fracture in the tip area of the stress concentrator. The fracture process in composites can have a various proceed, and the resulting cracks can propagate in miscellaneous directions.

Modelling and prediction of the fracture process is not particularly complicated if the stress concentrator is located inside one material phase. In such a situation, well known strength hypotheses used for homogeneous materials [1-4] can be applied to predict a composite strength. When the stress concentrator is somewhere else (e.g., on the interface), an adequate strength criterion must be formulated to predict fracture toughness. The criterion should contain a precisely defined equation with defined parameters, on the basis of which it is possible to predict the moment of initiating the fracture process. Criterion formulation usually requires knowledge of the description of the singular stresses occurring near the tip of the stress concentrator. Many scientists have dealt with singular stress fields. Some problems, for example, the interface crack [5;6], the structural notch [7] or the inclusion on the interface [8] are well investigated in the literature. Other issues, e.g., a notch, which tip is located on the interface have not been investigated sufficiently. Nevertheless, in the literature one can find the results of research for the special case of such a notch (with zero apex angle) - the crack starting at the interface [9-15]. For such a crack, assuming that it is perpendicular to the interface, the authors of [9] analysed the stress singularities occurring in its tip region. In [10] the eigenequation is presented and generalized stress intensity factors (GSIF) for the case of the short crack located in bi-material of infinite dimensions were determined. For the case of a crack located in an element with finite dimensions, the GSIFs were determined by the authors of the work [11-15].

As already mentioned, in composite materials there may also be a notch with the tip located on the interface, which tip angle is different from zero. Such a problem has not been sufficiently well studied in the literature. Thus, the main goal of the presented work is to obtain a qualitative and quantitative description of the singular stress fields occurring in the tip region of such a stress concentrator. Therefore, the first part of the work presents an analytical description of singular stress fields and the method of obtaining them. The quantitative description, associated with the calculation of GSIFs (for various geometric and material features of the composite), is presented in the second part of the paper. 


\section{Materials and methods}

In subsection 1, analytical solutions are given, and the method of their determination is discussed. The description of the procedures for determining GSIFs is presented in subsection 2. The third subsection discusses the numerical modelling carried out using the finite element method (FEM).

\section{Analytical solutions}

The eigenfunction expansion method was used to obtain an analytical description of the singular stress fields occurring in the tip area of the notch which apex is located on the interface (Fig. 1).

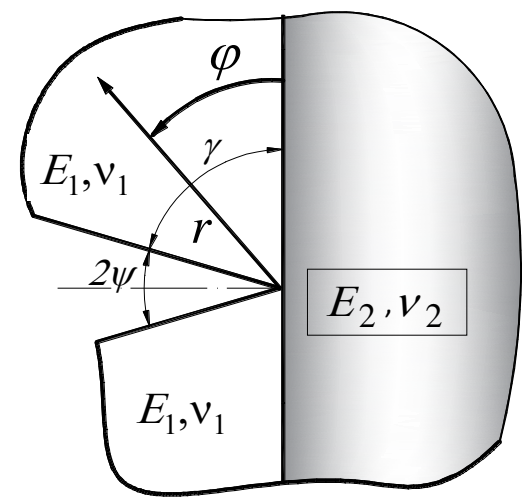

Fig. 1. Fragment of bi-material structure with a sharp corner, the top of which is located on the interface

A detailed description of this method can be found, for example, in works [16,17]. In the applied method, using the basic equations of the theory of elasticity and the Airy stress function, at the beginning general asymptotic solutions describing the individual components of stress and displacement fields are obtained. For the analysed notch problem, the general solutions are as follows:

$$
\begin{aligned}
& u_{r i}=r^{\lambda_{j}}\left(A_{i} \cos \left(\left(1+\lambda_{j}\right) \varphi\right)+B_{i} \sin \left(\left(1+\lambda_{j}\right) \varphi\right)+C_{i} \cos \left(\left(1-\lambda_{j}\right) \varphi\right)+D_{i} \sin \left(\left(1-\lambda_{j}\right) \varphi\right)\right) \\
& u_{\varphi i}=r^{\lambda_{j}}\left(-A_{i} \sin \left(\left(1+\lambda_{j}\right) \varphi\right)+B_{i} \cos \left(\left(1+\lambda_{j}\right) \varphi\right)-C_{i} \frac{\kappa_{i}+\lambda_{j}}{\kappa_{i}-\lambda_{j}} \sin \left(\left(1-\lambda_{j}\right) \varphi\right)+D_{i} \frac{\kappa_{i}+\lambda_{j}}{\kappa_{i}-\lambda_{j}} \cos \left(\left(1-\lambda_{j}\right) \varphi\right)\right) \\
& \left.\sigma_{r i}=r^{\lambda_{j}-1} \mu_{i}\left(A_{i} 2 \lambda_{j} \cos \left(\left(1+\lambda_{j}\right) \varphi\right)+B_{i} 2 \lambda_{j} \sin \left(\left(1+\lambda_{j}\right) \varphi\right)+C_{i}\left(3-\lambda_{j}\right) \frac{2 \lambda_{j}}{\kappa_{i}-\lambda_{j}} \cos \left(\left(1-\lambda_{j}\right) \varphi\right)+D_{i}\left(3-\lambda_{j}\right) \frac{2 \lambda_{j}}{\kappa_{i}-\lambda_{j}} \sin \left(\left(1-\lambda_{j}\right) \varphi\right)\right)\right\} . \\
& \sigma_{\varphi i}=r^{\lambda_{j}-1} \mu_{i}\left(-A_{i} 2 \lambda_{j} \cos \left(\left(1+\lambda_{j}\right) \varphi\right)-B_{i} 2 \lambda_{j} \sin \left(\left(1+\lambda_{j}\right) \varphi\right)+C_{i}\left(1+\lambda_{j}\right) \frac{2 \lambda_{j}}{\kappa_{i}-\lambda_{j}} \cos \left(\left(1-\lambda_{j}\right) \varphi\right)+D_{i}\left(1+\lambda_{j}\right) \frac{2 \lambda_{j}}{\kappa_{i}-\lambda_{j}} \sin \left(\left(1-\lambda_{j}\right) \varphi\right)\right) \\
& \tau_{r \varphi i}=r^{\lambda_{j}-1} \mu_{i}\left(-A_{i} 2 \lambda_{j} \sin \left(\left(1+\lambda_{j}\right) \varphi\right)+B_{i} 2 \lambda_{j} \cos \left(\left(1+\lambda_{j}\right) \varphi\right)+C_{i}\left(1-\lambda_{j}\right) \frac{2 \lambda_{j}}{\kappa_{i}-\lambda_{j}} \sin \left(\left(1-\lambda_{j}\right) \varphi\right)-D_{i}\left(1-\lambda_{j}\right) \frac{2 \lambda_{j}}{\kappa_{i}-\lambda_{j}} \cos \left(\left(1-\lambda_{j}\right) \varphi\right)\right)
\end{aligned}
$$

where $\quad \mu_{i}=\frac{E_{i}}{2\left(1+v_{i}\right)}-$ shear modulus;

$\kappa_{i}=\left(3-v_{i}\right) /\left(1+v_{i}\right)-$ plane stress;

$\kappa_{i}=\left(3-4 v_{i}\right)-$ plane strain;

$v_{i}$ - Poisson's ratio, $i=1,2$;

$j=I$ for symmetric problems (Mode I);

$j=I I$ for skew-symmetric problems (Mode II).

Eigenvalue $\lambda_{j}$ and constants $A_{i}, B_{i}, C_{i}, D_{i}$ are determined on the basis of boundary conditions. For the analysed problem (Figure 1), the boundary conditions were defined as follows.

1. Along the interface, for $\varphi=0$;

$$
u_{r 1}=u_{r 2} ; u_{\varphi 1}=u_{\varphi 2} ; \sigma_{\varphi 1}=\sigma_{\varphi 2} ; \tau_{r \varphi 1}=\tau_{r \varphi 2} \text {, }
$$

2. Of upper surface of V-notch, for $\varphi=\gamma$;

$$
\sigma_{\varphi 1}=\tau_{r \varphi 1}=0
$$


3. For $\varphi=-\pi / 2$;

- $\quad$ symmetry conditions (Mode I)

$\tau_{r \varphi 2}=u_{\varphi 2}=0$;

- $\quad$ skew-symmetry conditions (Mode II)

$$
\sigma_{\varphi 2}=u_{r 2}=0 \text {. }
$$

From the condition of zeroing the determinant of the matrix boundary conditions, eigenequations ((2)-Mode I, (3)-Mode II) were determined, which subsequent roots determine the values of eigenvalues $\lambda_{j}$ in the asymptotic solutions.

$$
\begin{aligned}
& \beta \lambda_{I} \sin [2 \gamma]+\left(\beta-1+2 \alpha\left(-1-\alpha+\beta+(\alpha-\beta) \lambda_{I}{ }^{2}\right)+2 \alpha(\beta-\alpha) \lambda_{I}{ }^{2} \cos [2 \gamma]\right) \sin \left[\pi \lambda_{I}\right]+ \\
& +\alpha(1+\alpha-\beta) \sin \left[(\pi-2 \gamma) \lambda_{I}\right]+(1+\alpha)(\alpha-\beta) \sin \left[(\pi+2 \gamma) \lambda_{I}\right]=0, \\
& \beta \lambda_{I I} \sin [2 \gamma]+\left(1-\beta+2 \alpha\left(1+\alpha-\alpha \lambda_{I I}{ }^{2}+\beta\left(\lambda_{I I}{ }^{2}-1\right)\right)+2 \alpha(\alpha-\beta) \lambda_{I I}{ }^{2} \cos [2 \gamma]\right) \sin \left[\pi \lambda_{I I}\right]+ \\
& +\alpha(\beta-\alpha-1) \sin \left[(\pi-2 \gamma) \lambda_{I I}\right]-(1+\alpha)(\alpha-\beta) \sin \left[(\pi+2 \gamma) \lambda_{I I}\right]=0 .
\end{aligned}
$$

where $\quad \alpha=\frac{\mu_{1} / \mu_{2}-1}{\left(1+\kappa_{1}\right)}, \beta=\frac{\mu_{1}\left(1+\kappa_{2}\right)}{\mu_{2}\left(1+\kappa_{1}\right)}$.

It is worth noting that in adopting the equations (2) and (3) the following assumptions$\alpha=0, \beta=1, \gamma=\gamma-\pi / 2$ - eigenequations obtained are identical as for the notch problem in homogeneous material [16]:

$$
\begin{aligned}
& \lambda_{I} \sin [2 \gamma]+\sin \left[2 \gamma \lambda_{I}\right]=0, \\
& \lambda_{I I} \sin [2 \gamma]-\sin \left[2 \gamma \lambda_{I}\right]=0 .
\end{aligned}
$$

The calculations showed that regardless of the material constants and notch angle, the roots of equations (2) and (3) $-\lambda_{j}$ - always assume real values (in the range of $0 \div 1$ ). In addition, the singularities of stress fields increase monotonically ( $\lambda_{j}$ assumes smaller values) as the proportion $\mu_{1} / \mu_{2}$ increases and the notch angle decreases. Exemplary solutions of equations (7), for arbitrarily assumed notch angles, are shown graphically in Figure 2.

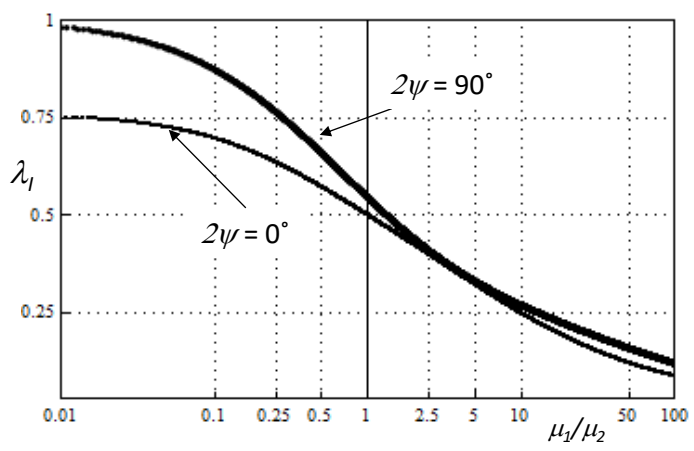

Fig. 2. Solution of eigenequations $\left(v_{1}=v_{2}=0.3\right.$, plane stress condition)

As for the analytical description of the stress fields, in order to avoid a trivial solution $\left(A_{i}, B_{i}, \ldots=0\right)$, the matrix of boundary conditions had to be modified. The modification consisted in replacing two arbitrarily selected boundary conditions with adopted definitions of the stress intensity factors. Since eigenvalues $\lambda_{j}$ always assume real values, the generalized stress intensity factors $K_{j}$ were defined, similarly to the work [16], as follows:

$$
\left.\begin{array}{l}
K_{I}=\lim _{r \rightarrow 0} \sqrt{2 \pi} r^{1-\lambda_{l}} \sigma_{\varphi 2}(r,-\pi / 2) \\
K_{I I}=\lim _{r \rightarrow 0} \sqrt{2 \pi} r^{1-\lambda_{I I}} \tau_{r \varphi 2}(r,-\pi / 2)
\end{array}\right\} .
$$

After applying thus obtained constants $A_{i}, B_{i}, C_{i}, D_{i}$ to formula (6), an analytical description of stress fields was obtained. Because the fracture process will most likely run along the interface or in material 2 , only the formulas describing stresses in material 2 are given below. 


$$
\begin{aligned}
& \sigma_{\varphi 2}=\left(\begin{array}{l}
\frac{-K_{I} r^{\lambda_{I}-1}\left(\left(1+\lambda_{I}\right) \cos \left[\frac{1}{2}\left(\lambda_{I}-1\right)(\pi+2 \varphi)\right] F_{I 2}+\alpha \mu_{l}\left(\lambda_{I}-\kappa_{2}\right) \sin \left[\frac{\pi \lambda_{I}}{2}+\varphi+\lambda_{I} \varphi\right] F_{I 1}\right)}{\sqrt{2 \pi} \alpha \mu_{1}\left(\lambda_{I}-\kappa_{2}\right) F_{I 0}}+ \\
\frac{K_{I I} r^{\lambda_{I I}-1}\left(\left(1+\lambda_{I I}\right) \cos \left[\frac{\pi \lambda_{I I}}{2}+\left(\lambda_{I I}-1\right) \varphi\right] F_{I I 2}+\alpha\left(\lambda_{I I}-\kappa_{2}\right) \cos \left[\frac{\pi \lambda_{I I}}{2}+\varphi+\lambda_{I I} \varphi\right] F_{I I 1}\right)}{\sqrt{2 \pi} \alpha \mu_{1}\left(\lambda_{I I}-\kappa_{2}\right) F_{I I 0}}
\end{array}\right) \\
& \tau_{r \varphi 2}=\left(\begin{array}{l}
\frac{K_{I} r^{\lambda_{I}-1}\left(\left(\lambda_{I}-1\right) \cos \left[\frac{\pi \lambda_{I}}{2}+\left(\lambda_{I}-1\right) \varphi\right] F_{I 2}+\alpha \mu_{1}\left(\lambda_{I}-\kappa_{2}\right) \cos \left[\frac{\pi \lambda_{I}}{2}+\varphi+\lambda_{I} \varphi\right] F_{I 1}\right)}{\sqrt{2 \pi} \alpha \mu_{1}\left(\lambda_{I}-\kappa_{2}\right) F_{I 0}}+ \\
+\frac{K_{I I} r^{\lambda_{I I}-1}\left(\left(\lambda_{I I}-1\right) \cos \left[\frac{1}{2}\left(\lambda_{I I}-1\right)(\pi+2 \varphi)\right] F_{I I 2}+\alpha\left(\lambda_{I I}-\kappa_{2}\right) \sin \left[\frac{\pi \lambda_{I I}}{2}+\varphi+\lambda_{I I} \varphi\right] F_{I I 1}\right)}{\sqrt{2 \pi} \alpha \mu_{1}\left(\lambda_{I I}-\kappa_{2}\right) F_{I I 0}}
\end{array}\right) \\
& \sigma_{r 2}=\left(\begin{array}{l}
\frac{K_{I} r^{\lambda_{I}-1}\left(\left(\lambda_{I}-3\right) \cos \left[\frac{1}{2}\left(\lambda_{I}-1\right)(\pi+2 \varphi)\right] F_{I 2}+\alpha \mu_{1}\left(\lambda_{I}-\kappa_{2}\right) \sin \left[\frac{\pi \lambda_{I}}{2}+\varphi+\lambda_{I} \varphi\right] F_{I 1}\right)}{2 \sqrt{2 \pi} \alpha \mu_{1}\left(\lambda_{I}-\kappa_{2}\right) F_{I 0}}+ \\
\left.-\frac{K_{I I} r^{\lambda_{I I}-1}\left(\left(\lambda_{I I}-3\right) \cos \left[\frac{\pi \lambda_{I I}}{2}+\left(\lambda_{I I}-1\right) \varphi\right] F_{I I 2}+\alpha\left(\lambda_{I I}-\kappa_{2}\right) \cos \left[\frac{\pi \lambda_{I I}}{2}+\varphi+\lambda_{I I} \varphi\right] F_{I I 1}\right)}{\sqrt{2 \pi} \alpha \mu_{1}\left(\lambda_{I I}-\kappa_{2}\right) F_{I I 0}}\right)
\end{array}\right)
\end{aligned}
$$

where

$$
\begin{aligned}
F_{I 1}= & \begin{array}{l}
-\alpha\left(\lambda_{I}-1\right) \lambda_{I} \cos \left[\gamma+\frac{\pi \lambda_{I}}{2}-\gamma \lambda_{I}\right]+\left(1+\alpha-\beta+\alpha \lambda_{I}^{2}\right) \cos \left[\gamma-\frac{\pi \lambda_{I}}{2}+\gamma \lambda_{I}\right]+ \\
\left.+(\beta-2 \alpha-1) \lambda_{I} \cos \left[\gamma+\frac{\pi \lambda_{I}}{2}+\gamma \lambda_{I}\right]+(\alpha-\beta)\left(\lambda_{I}-1\right) \cos \left[\gamma-\frac{1}{2}(\pi+2 \gamma) \lambda_{I}\right]\right),
\end{array} \\
F_{I 2}= & \left.\left(\alpha-\beta+\alpha \lambda_{I}\right) \mu_{1}+\beta \mu_{2}\right)\left(\cos \left[\gamma+\frac{\pi \lambda_{I}}{2}+\gamma \lambda_{I}\right]-2 \alpha\left(\lambda_{I} \sin [\gamma] \sin \left[\frac{1}{2}(\pi-2 \gamma) \lambda_{I}\right]+\sin \left[\gamma \lambda_{I}\right] \sin \left[\gamma+\frac{\pi \lambda_{I}}{2}\right]\right)\right), \\
F_{I 0}= & \left.\begin{array}{l}
2 \cos [\gamma]\left(-\lambda_{I} \cos \left[\frac{\pi \lambda_{I}}{2}\right] \cos \left[\gamma \lambda_{I}\right]+\left(1-\beta+\lambda_{I}+2 \alpha\left(1+\lambda_{I}\right)\right) \sin \left[\frac{\pi \lambda_{I}}{2}\right] \sin \left[\gamma \lambda_{I}\right]\right)+ \\
+\sin [\gamma]\left(\left(1-\beta+4 \alpha \lambda_{I}^{2}\right) \sin \left[\frac{1}{2}(\pi-2 \gamma) \lambda_{I}\right]+\left(1+\beta+2 \lambda_{I}+4 \alpha \lambda_{I}-2 \beta \lambda_{I}\right) \sin \left[\frac{1}{2}(\pi+2 \gamma) \lambda_{I}\right]\right)
\end{array}\right), \\
F_{I I 1}= & \left.\begin{array}{l}
\alpha \lambda_{I I}\left(1+\lambda_{I I}\right) \cos \left[\gamma+\frac{\pi \lambda_{I I}}{2}-\gamma \lambda_{I I}\right]-\left(1+\alpha-\beta+\alpha \lambda_{I I}^{2}\right) \cos \left[\gamma-\frac{\pi \lambda_{I I}}{2}+\gamma \lambda_{I I}\right]+ \\
\left.(\beta-1-2 \alpha) \lambda_{I I} \cos \left[\gamma+\frac{\pi \lambda_{I I}}{2}+\gamma \lambda_{I I}\right]+(\alpha-\beta)\left(1+\lambda_{I I}\right) \cos \left[\gamma-\frac{1}{2}(\pi+2 \gamma) \lambda_{I I}\right]\right)
\end{array}\right) \\
F_{I I 2}= & \left.(\alpha-\beta+\alpha \lambda) \mu_{1}+\beta \mu_{2}\right)\left(\begin{array}{l}
(1+\alpha) \cos \left[\gamma+\frac{\pi \lambda_{I I}}{2}+\gamma \lambda_{I I}\right]+ \\
\left.+\alpha \lambda_{I I} \cos \left[\gamma-\frac{\pi \lambda_{I I}}{2}+\gamma \lambda_{I I}\right]-\alpha\left(1+\lambda_{I I}\right) \cos \left[\gamma+\frac{\pi \lambda_{I I}}{2}-\gamma \lambda_{I I}\right]\right)
\end{array}\right) \\
F_{I I 0}= & \left(\begin{array}{l}
2 \cos [\gamma]\left(\lambda_{I I} \cos \left[\frac{\pi \lambda_{I I}}{2}\right] \cos \left[\gamma \lambda_{I I}\right]-\left(\beta-1+2 \alpha\left(\lambda_{I I}-1\right)+\lambda_{I I}\right) \sin \left[\frac{\pi \lambda_{I I}}{2}\right] \sin \left[\gamma \lambda_{I I}\right]\right)+ \\
+\sin [\gamma]\left(\left(1-\beta+4 \alpha \lambda_{I I}^{2}\right) \sin \left[\frac{1}{2}(\pi-2 \gamma) \lambda_{I I}\right]+\left(1+\beta+2(\beta-1-2 \alpha) \lambda_{I I}\right) \sin \left[\frac{1}{2}(\pi+2 \gamma) \lambda_{I I}\right]\right)
\end{array}\right) .
\end{aligned}
$$

For the quantitative description of stresses it is necessary to determine the values of the $K_{j}$ coefficients. They were determined using the approach proposed in [18]. The idea of the applied method of determining generalized stress intensity factors, along with the necessary modifications enabling its use for a sharp notch with the tip on the interface, is discussed below.

\section{The method of determining generalized stress intensity factors $\boldsymbol{K}_{\boldsymbol{j}}$}

As already mentioned, the quantitative description of singular stress fields consists in determining generalized stress intensity factors $K_{j}(\mathrm{GSIF})$. The exact solution, enabling the determination of the GSIF, is known only for the case of a crack perpendicular to the interface situated in a bi-material 
structure with infinite dimensions [10]. For sharp corners with the tip located on the interface, with different geometrical features, approximate methods should be used to determine the generalised stress intensity factors. The presented work uses the analytical and numerical method [18]. In order to use it, it is necessary to know the analytical description of stress fields, on the basis of which specific analytical formulas describing the GSIFs at a certain distance from the notch tip are formed, as well as data from numerical tests. The procedures related to numerical modelling using the finite element method (FEM) are discussed in the next section. In this part of the work, the specific analytical formulas used to determine the GSIF are derived.

Using the equation (5), the hoop and tangential stresses at a distance $r_{n}$ and $r_{n+1}$ from the notch tip can be written as follows:

$$
\left.\begin{array}{c}
\sigma_{\varphi 2\left(r_{n},-\pi / 2\right)}=\frac{K_{I(r)}}{\sqrt{2 \pi} r^{1-\lambda_{I}}}\left(1+c_{I} r_{n}\right), \sigma_{\varphi 2\left(r_{n}+1,-\pi / 2\right)}=\frac{K_{I(r)}}{\sqrt{2 \pi} r^{1-\lambda_{I}}}\left(1+c_{I} r_{n+1}\right) \\
\tau_{r \varphi 2\left(r_{n},-\pi / 2\right)}=\frac{K_{I I(r)}}{\sqrt{2 \pi} r^{1-\lambda_{I I}}}\left(1+c_{I I} r_{n}\right), \tau_{r \varphi 2\left(r_{n}+1,-\pi / 2\right)}=\frac{K_{I I(r)}}{\sqrt{2 \pi} r^{1-\lambda_{I I}}}\left(1+c_{I I} r_{n+1}\right)
\end{array}\right\} .
$$

On the basis of the above formulas (7), after simple mathematical transformations, linear functions are obtained, which enable the determination of the required general stress intensity factors $K_{j(r)}$ (at a certain distance from the notch tip):

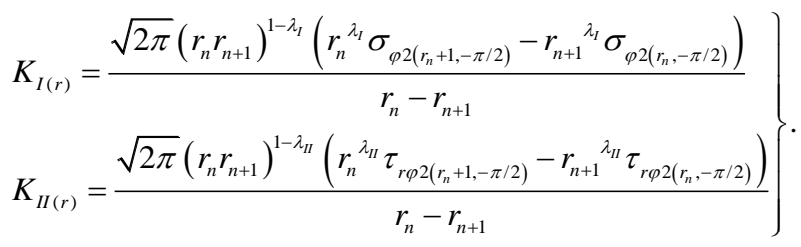

The $K_{j(r)}$ factors are calculated using the appropriate stresses obtained from FEM in formula (8). As it is commonly known, in analytical and numerical methods the accuracy of the obtained results depends largely on the selection of the area, from which the data obtained from the FEM solution are applied to the developed analytical functions. In this paper, the criterion presented in [18] was used to define such an area.

Thus, the calculated coefficients for all $n+1$ nodes should be theoretically identical. However, due to potential errors in numerical calculations, the results may differ slightly. Therefore, in order to minimize the error, the obtained results can be averaged according to the equation (9):

$$
K_{I}=\frac{\sum_{n=1}^{n+1} K_{I(r)}}{n+1}, K_{I I}=\frac{\sum_{n=1}^{n+1} K_{I I(r)}}{n+1} .
$$

In order to verify the method used, the $K_{j}$ coefficients were calculated for the case of a crack initiated at the interface of the bi-material structure. The obtained results were compared with the exact solution [10] and the approximate one [19]. A satisfactory agreement was obtained in both cases - the difference was about $1.2 \%$.

\section{FEM modelling}

With the development of numerical methods, nowadays, a FEM-based numerical approach is very often used. FEM, combined with analytical or experimental solutions, can be used, e.g., to study phenomena such as friction [20;21] liquid and heat flow [22;23], or piezoelectricity [24;25]. This method can also be useful to determine generalized stress intensity factors. Therefore, the bi-material structure, with a sharp notch, the tip of which is located on the interface, was modelled with the use of ANSYS Environment (Mechanical APDL). The specimens with a single edge sharp corner under uniaxial tension (symmetric problems (Mode I)) were modelled in numerical simulations (Fig.3a). 


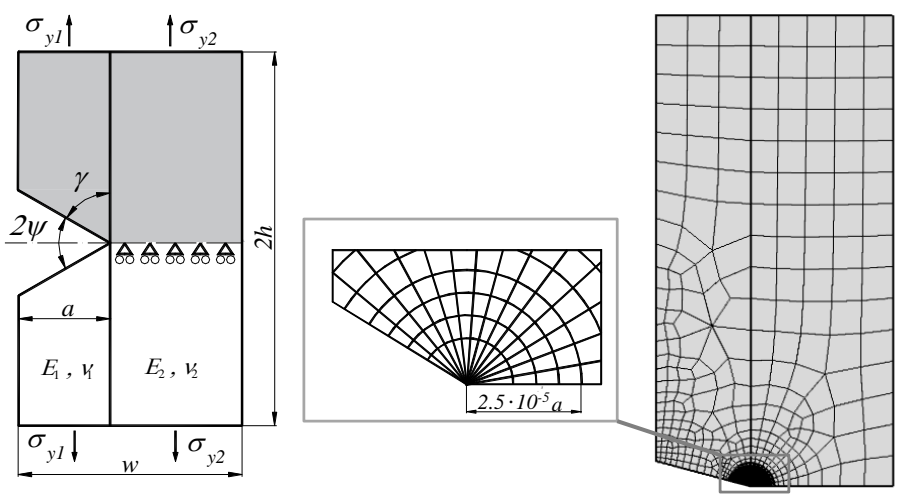

a) b)

Fig. 3. Specimens with a single edge-notched plate: a - geometry and method of fixing and loading, $\mathrm{b}$ - typical finite element mesh

Due to the symmetry of the specimens, their halves were modelled (the area marked in grey in Figure 3a). Symmetry boundary conditions were assumed in the symmetry planes. The specimens are described with a mesh of quadrangular eight-node finite elements of an increased concentration around the tip area (Fig. 3b).

In all simulations, the specimens were loaded with a constant load of $\sigma_{y 1}=1 \mathrm{~Pa}$, assuming a plane stress condition. The value of the load $\sigma_{y 2}$ was determined on the basis of the formula (10), resulting from the condition of continuity of strain $-\varepsilon_{y 1}=\varepsilon_{y 2}$ :

$$
\sigma_{y 2}=\frac{E_{2}\left(\sigma_{y 1}-\sigma_{x} v_{1}\right)}{E_{1}}+\sigma_{x} v_{2} .
$$

The simulations were prepared for various relative stiffness of the individual components of the bimaterial:

$$
\Gamma=\mu_{1} / \mu_{2},
$$

where $\mu_{i}=\frac{E_{i}}{2\left(1+v_{i}\right)}$.

In all simulations, it was assumed that the Young's modulus of material 1 was constant and be equal to: $E_{1}=1 \cdot 10^{9} \mathrm{~Pa}$. On the other hand, the Young's modulus of material 2- $E_{2}-$ was variable and depended on the parameter $\Gamma$. The Poisson coefficients also depended on this parameter, which were respectively: $v_{1}=v_{2}=0.3$ (for $\left.\Gamma=1\right), v_{1}=0.3, v_{2}=0.35$ (for $\Gamma>1$ ), and $v_{1}=0.35, v_{2}=0.3$ (for $\Gamma<1$ ).

As for the geometrical dimensions, the notched specimens with variable apex angle $\psi$ were modelled. Moreover, in the numerical models, the ratio of the notch height to the specimen width- $a / w$ was also changed, with the notch height $a$ being constant $-a=1 \mathrm{~m}$. In all simulations the proportion of the remaining linear dimensions was kept constant $-h / w=2$.

\section{Results and discussion}

In this part of the work, the generalized stress intensity factors calculated for the samples described in previous section are presented. To calculate the $K_{I}$ coefficients (for tensile specimens, the $K_{I I}$ coefficients are always equal to zero), it was necessary to determine the eigenvalues $\lambda_{I}$. The values of $\lambda_{l}$, calculated on the basis of formulas (2), are presented in Table 1.

Due to the dimensions for the GSIF's $\left[\mathrm{Pa} \cdot \mathrm{m}^{1-\lambda j}\right]$, it is difficult to compare the results obtained. In such a situation, their normalized values are most often used [13]. Thus, the determined GSIFs were normalized according to the formula below:

$$
F_{j}=K_{j} /\left(\sigma_{y 1} \sqrt{\pi} a^{1-\lambda_{j}}\right)
$$

Table 2 shows the normalized $F_{I}$ factors calculated for the specimens with various relative stiffness and notch apex angles. 
Values of eigenvalues $\lambda_{I}$ for specimens subjected to tensile loads

Table 1

\begin{tabular}{|c|c|c|l|l|l|l|l|l|}
\hline $2 \psi$ & $\gamma$ & \multicolumn{7}{|c|}{$\lambda_{I}$} \\
\cline { 3 - 9 } & $\Gamma=0.1$ & $\Gamma=0.2$ & $\Gamma=0.5$ & $\Gamma=1$ & $\Gamma=2$ & $\Gamma=5$ & $\Gamma=10$ \\
\hline$\left.\Gamma^{\circ}\right]$ & 90 & 0.681447 & 0.640755 & $\begin{array}{c}0.563832 / \\
0\end{array}$ & 0.5 & $\begin{array}{c}0.42944 / 0 . \\
42944 *\end{array}$ & 0.32579 & 0.25150 \\
& & & $0.56383^{*}$ & & & \\
\hline 30 & 75 & 0.729362 & 0.671115 & 0.573859 & 0.50145 & 0.43042 & 0.33514 & 0.26647 \\
\hline 60 & 60 & 0.795359 & 0.720259 & 0.599749 & 0.51222 & 0.43166 & 0.33627 & 0.27426 \\
\hline 90 & 45 & 0.866117 & 0.786945 & 0.649842 & 0.54448 & 0.44639 & 0.33606 & 0.27385 \\
\hline
\end{tabular}

Values of normalized stress intensity factors $F_{I}, a / w=0.2$

Table 2

\begin{tabular}{|c|c|c|c|c|}
\hline \multirow{2}{*}{$\Gamma$} & \multicolumn{4}{|c|}{$F_{I}$} \\
\cline { 2 - 5 } & $2 \psi=0^{\circ}$ & $2 \psi=30^{\circ}$ & $2 \psi=60^{\circ}$ & $2 \psi=90^{\circ}$ \\
\hline 0.1 & 4.317 & 6.070 & 8.765 & 11.990 \\
\hline 0.2 & 3.076 & 3.722 & 4.713 & 5.991 \\
\hline 0.5 & 1.908 & 2.019 & 2.236 & 2.609 \\
\hline \multirow{2}{*}{1} & 1.361 & 1.375 & 1.431 & 1.579 \\
\cline { 2 - 5 } & $1.367 *$ & & $2.230 * / 2.220^{* *}$ & $2.478^{*} / 2.471^{* *}$ \\
\hline 2 & 0.983 & 0.983 & 0.989 & 1.042 \\
\hline 5 & 0.636 & 0.659 & 0.659 & 0.659 \\
\hline 10 & 0.452 & 0.490 & 0.502 & 0.504 \\
\hline \multicolumn{6}{|c|}{$*$-From reference [26], **-calculated for $a / w=0.4$} \\
\hline
\end{tabular}

On the basis of the obtained results, it can be concluded that the normalized values of the generalized stress intensity factors $F_{I}$ increase with the decrease of the relative stiffness $\Gamma$. This tendency is consistent with the distribution of the $F_{I}$ coefficients for the problem of a crack oriented transversely to the interface of the two-phase structure, with the tip reaching the boundary of both materials $[10 ; 19]$ The value of the stress intensity factors is also affected by the notch angle $2 \psi . F_{I}$ factors increase along with its increase. It is worth noting that the influence of the $\psi$ angle on the $F_{I}$ coefficients is more significant for cases when $\Gamma<1$. The study also investigated the effect of the notch height on the values of the GSIFs. As expected, an increase in the notch height, regardless of the material parameters and the apex angle, always increases the value of the $F_{I}$ coefficients (Fig. 5).

a)

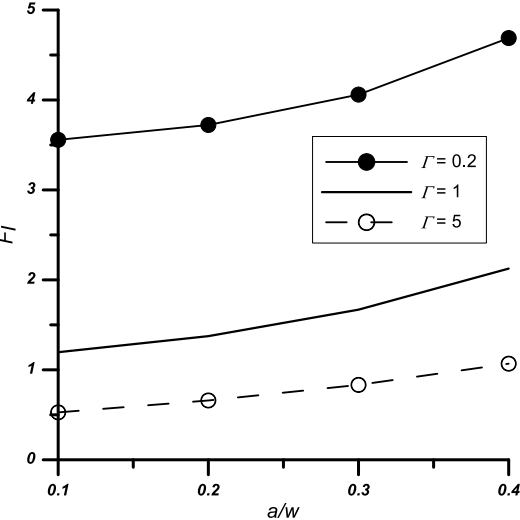

b)

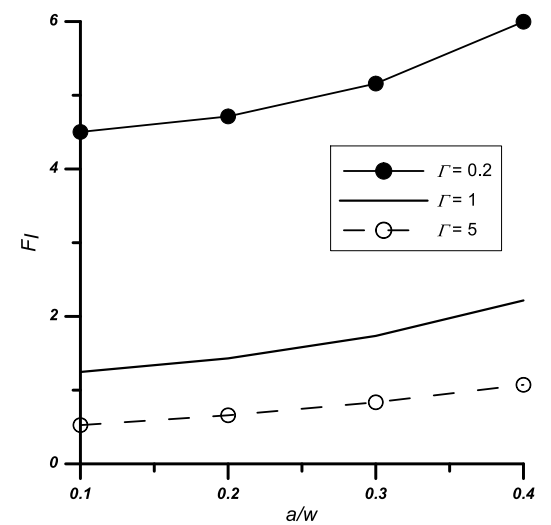

Fig. 5. Distribution of $\boldsymbol{F}_{I}$ coefficients depending on the relative stiffness $\Gamma$ and notch height $a / w$ : a-notched element with apex angle $2 \psi=30^{\circ}$, b-notched element with apex angle $2 \psi=60^{\circ}$

\section{Conclusions}

1. For all load cases, the eigenequations depend on the material constants of the bi-material and the notch tip angle.

2. Stress fields, regardless of the type of load, have single real singularities. 
3. The normalized values of the generalized stress intensity factors $F_{I}$ increase with a decrease in the relative stiffness $\Gamma$ and an increase in the notch height and its apex angle.

4. A satisfactory agreement was found with the results presented by other scientists-relative error less than $1.5 \%$.

\section{Acknowledgements}

This research was founded through subsidy of the Ministry of Science and Higher Education of Poland for the discipline of mechanical engineering at the Faculty of Mechanical Engineering, Bialystok University of Technology WZ/WM-IIM/4/2020.

\section{Funding}

This publication was financed through the program of the Ministry of Science and Higher Education of Poland named "Regional Initiative of Excellence" in 2019-2022 project number 011/RID/2018/19.

\section{References}

[1] Griffits A.A. The phenomena of rupture and flow in solids. Philosophical Transactions of the Royal Society of London Series A, Containing Papers of a Mathematical or Physical Character, vol. 221, 1921, pp. 163-98.

[2] Sih G.C. Strain-energy-density factor applied to mixed mode crack problems. International Journal of Fracture, vol.10, 1974, pp. 305-21.

[3] McClintock F.A. Ductile fracture instability in shear. Journal of Applied Mechanics, vol. 25, 1958,pp. 582-588.

[4] Yosibash Z., Priel E., Leguillon D. A failure criterion for brittle elastic materials under mixed-mode loading. International Journal of Fracture, vol. 141, 2006, pp. 291-312.

[5] Sih G.C, Chen E.P. Cracks in materials possessing homogeneous anisotropy. Cracks in composite materials, Springer Netherlands, 1981, p. 1-101.

[6] Sun C.T., Jih C.J. On strain energy release rates for interfacial cracks in bi-material media. Engineering Fracture Mechanics,vol. 28, 1987, pp. 13-20.

[7] Krishnan A., Roy Xu L. An experimental study on the crack initiation from notches connected to interfaces of bonded bi-materials. Engineering Fracture Mechanics, vol. 111, 2013, pp. 65-76.

[8] Ballarini R. A rigid line inclusion at a bimaterial interface. Engineering Fracture Mechanics, vol. 37, 1990, pp. 1-5.

[9] Zak A.R., Williams M.L. Crack point stress singularities at a bi-material interface. Journal of Applied Mechanics, vol. 30, 1960, pp. 142-143.

[10] Cook T.S., Erdogan F. Stresses in bonded materials with a crack perpendicular to the interface. International Journal of Engineering Science, vol. 10, 1972, pp. 677-697.

[11] Wang W.C, Chen J.T. Theoretical and experimental re-examination of a crack perpendicular to and terminating at the bimaterial interface. The Journal of Strain Analysis for Engineering Design, vol. 28, 1993, pp. 53-61.

[12] Meguid S.A, Tan M., Zhu Z.H. Analysis of cracks perpendicular to bimaterial interfaces using a novel finite element. International Journal of Fracture, vol. 73, 1985,pp. 1-23.

[13] Dai-Heng C. A crack normal to and terminating at a bimaterial interface. Engineering Fracture Mechanics, vol. 49, 1994, pp. 517-532.

[14] Keikhaie M., Keikhaie N., Keikhaie R., Kaykha M.M. Stress Intensity Factors in Two Bonded Elastic Layers Containing Crack Perpendicular on the Interface with Different Elastic Properties. Journal of Modern Physics, vol. 06, 2015, pp. 640-647.

[15] Chang J., Xu J.Q. The singular stress field and stress intensity factors of a crack terminating at a bimaterial interface. International Journal of Mechanical Sciences, vol. 49, 2007, pp. 888-897.

[16] Williams M.L. Stress Singularities Resulting from Various Boundary Conditions in Angular Corners of Plates in Extension. Journal of Applied Mechanics, vol. 19, 1952, pp. 526-528.

[17] Parton V.Z, Perlin P.I. Mathematical Methods of the Theory of Elasticity. Moscow: Mir Publishers, 1984. $356 \mathrm{p}$. 
[18] Mieczkowski G. Determination of stress intensity factors for elements with sharp corner located on the interface of a bi-material structure or homogeneous material. Acta Mechanica, vol. 232, 2020, pp. 709-724.

[19] Lin K.Y, Mar J.W. Finite element analysis of stress intensity factors for cracks at a bi-material interface. International Journal of Fracture, vol. 12, 1976, pp. 521-31.

[20] Borawski A., Szpica D., Mieczkowski G. Verification tests of frictional heat modelling results. Mechanics, vol. 26, 2020, pp. 260-264.

[21] Łukaszewicz A. Nonlinear Numerical Model of Friction Heating during Rotary Friction Welding. Journal of Friction and Wear, vol. 39, 2018, pp. 476-482.

[22] Borawski A., Szpica D., Mieczkowski G., Borawska E., Awad M.M, Shalaby R.M, et al. Theoretical Analysis of the Motorcycle Front Brake Heating Process during High Initial Speed Emergency Braking. Journal of Applied and Computational Mechanics, vol. 6, 2020, pp. 1431-7.

[23] Yevtushenko A.A, Kuciej M., Grzes P., Wasilewski P.. Temperature in the railway disc brake at a repetitive short-term mode of braking. International Communications in Heat and Mass Transfer, vol. 84, 2017, pp. 102-109.

[24] Mieczkowski G. , Borawski A. , Szpica D. Static electromechanical characteristic of a three-layer circular piezoelectric transducer. Sensors, vol. 20, 2020, 222, 14p.

[25] Mieczkowski G. Static Electromechanical Characteristics of Piezoelectric Converters with various Thickness and Length of Piezoelectric Layers. Acta Mechanica et Automatica, vol. 13, 2019, pp. 30-36.

[26] Treifi M., Oyadiji S.O. Bi-material V-notch stress intensity factors by the fractal-like finite element method. Engineering Fracture Mechanics, vol. 105, 2013, pp. 221-237 